\title{
OP63
}

\section{EXPERIMENT OF LIGHTING ENVIRONMENTS IN WARD FOR BLOOD CANCER PATIENTS BASED ON VR TECHNOLOGY}

\author{
Shao Rongdi et al.
}

DOI 10.25039/x46.2019.OP63

from

CIE x046:2019

Proceedings

of the

29th CIE SESSION

Washington D.C., USA, June 14 - 22, 2019

(DOI 10.25039/x46.2019)

The paper has been presented at the 29th CIE Session, Washington D.C., USA, June 14-22, 2019. It has not been peer-reviewed by CIE.

(C) CIE 2019

All rights reserved. Unless otherwise specified, no part of this publication may be reproduced or utilized in any form or by any means, electronic or mechanical, including photocopying and microfilm, without permission in writing from CIE Central Bureau at the address below. Any mention of organizations or products does not imply endorsement by the CIE.

This paper is made available open access for individual use. However, in all other cases all rights are reserved unless explicit permission is sought from and given by the CIE.

CIE Central Bureau

Babenbergerstrasse 9

A-1010 Vienna

Austria

Tel.: +43 17143187

e-mail: ciecb@cie.co.at

www.cie.co.at 


\title{
EXPERIMENT OF LIGHTING ENVIRONMENTS IN WARD FOR BLOOD CANCER PATIENTS BASED ON VR TECHNOLOGY
}

\author{
Shao, R.D. ${ }^{1,2}$, Feng, K. ${ }^{1,2}$, Ge, W.J. ${ }^{1,2}$, Hao, L.X. ${ }^{1,2}$ \\ 1 College of Architecture and Urban Planning, Tongji University, Shanghai, CHINA; \\ ${ }^{2}$ Key Laboratory of Ecology and Energy Saving Study of Dense Habitat (Tongji University), \\ Ministry of Education, CHINA \\ haoluoxi@tongji.edu.cn
}

DOI 10.25039/x46.2019.OP63

\begin{abstract}
The interior design of a hospital building should follow a scientific and rigorous evidence-based design research process; particularly, the feasibility should be continuously evaluated and modified during the interior design of the light environment in hospital, so as to avoid irreversible damage to patient during application. This study took the light and colour environment reform in the haematology department ward of China as an example, and employed the VR technology to conduct the evidence-based experimental research. Using the VR technology, scene adjustment of parameters such as colour temperature, brightness, light colour and interior decoration colour of the haematology ward could be achieved, and the scene evaluation factors of patients in the simulated real environment could be explored using the relevant factors, which would finally contribute to the formation of a set of favourable control scene parameters conducive to patient recovery.
\end{abstract}

Keywords: Evidence-based design, Blood Cancer Patient wards, Lighting Environment

\section{Introduction}

The design of the indoor environment of patient wards should be scientifically and rigorously designed according to the characteristics of its use and the particularity of the people in use. Evidence-based design originated from evidence-based medicine(Hamilton D. K., et al. , 2012), and the US Centre for Health Design defines evidence-based design as "a design process based on reliable research results that is based on informed decisions about the built environment to achieve optimal results." This requires design researchers to fully understand the user's needs, uses professional knowledge to analyse the user's needs, obtain evidence of reliability, and thus be able to guide the design practice. In the study of the process of personal spatial perception. It has been proved that the stimuli in the visual channel and the auditory channel all have effects, but the visual channel stimulation is the main criterion for judgment, and there is a visual priority effect (Zeng, Q., et al., 2018). Therefore, the light environment and colour environment play an important role in the indoor space as the main visual element.

The treatment of blood cancer is regularly daily examination and high requirements for fine visual operations such as drug sorting, injection and infusion. Patient should stay in bed for long-time transfusion, and they should strictly avoid knocking against or falling down. To avoid highly infectious risk, they should be isolated by laminar flow bed which is uncomfortable or wearing masks all the time. Medical staff have high pressure in their work treatment process of blood cancer; and the depression ratio in blood cancer patients is high. Compared with other patients, the negative emotional characteristics of haematology patients are obvious, which are manifested as suspicion, fear, depression and pessimistic despair, and even suicide. Patient ward requires of wall colour in soft hue, small psychological and physiological stimuli, such as light green, light yellow and light pink; high brightness to increase the openness of space and colour matching recommended high-short Tone (High: Brightness is high-short tone: The difference between maximum brightness and minimum brightness is less than 3 tones) to create an atmosphere of light and gentle colour(An, N.,1999.).

With the advent of VR technology, the way to obtain the best evidence for evidence-based design has been expanded. VR technology can create solutions that are far better than the 
traditional experimental methods in terms of use effect for users through the interpretation of different demands of use and the construction of different scenes, and promote relevant research and practical work. VR technology has been applied in military simulation, urban construction engineering, education, entertainment and other fields, and brings multiple conveniences. VR technology, by virtue of its two core features of hypertext and interactivity, provides users with incomparable advantages over traditional technology in terms of scene simulation and cognitive experience. VR technology has been very extensively applied in the medical field, and become more and more mature. VR technology has been applied in the realtime record of colon cancer tumour resection, total knee joint replacement and ophthalmic surgery (WU, Z. et al.,2017). VR technology is also applied for preoperative disease prognosis in the medical field, which can better ensure the smooth operation (QIU, H., et al.,2019). In addition, VR technology can help patients with physical recovery training and psychological intervention, and assist disabled people to improve their balance ability through VR simulation of rowing, playing ball, jogging and other scenarios; and intervene the psychotherapy of schizophrenic patients through the setting of specific scenes(HUANG, C., et al., 2019).

\section{Method}

\subsection{Tasks}

This research takes the ward of a triple-human blood cancer patient as an example, and the indoor simulation model of the existing ward is established by VR technology. The colour of soft hue and high short tune collocation is used as the ambient colour, and 3 possible lighting scene models are established in the pre-experiment. Through the built-in IES light parameters and RGB chromaticity parameters, VR enables precise simulation of brightness and chroma. The selection of 6 patients with haematological diseases and 6 medical staff to carry out formal experiments are required to evaluate the pre-set scene model through the scale.

\subsection{Place and Equipment}

The experiment place is located on the 7 th floor of haematology ward in the Internal Medicine building, The First Affiliated Hospital of Henan Science and Technology University of China. Because the haematological disease has many anaemia symptoms, mostly stay in bed for rest and they can't go out for experiments. Therefore the VR experiment implemented in the patients' long-term adaptation environment with the medical care protection, so that the experiment can be carried on more easily without delaying its treatment and rest.

VR Equipment: HTC VIVE Pro with technology of Steam VR tracking technology, G-sensor calibration, gyroscope, proximity distance sensor, and Eye distance sensor.

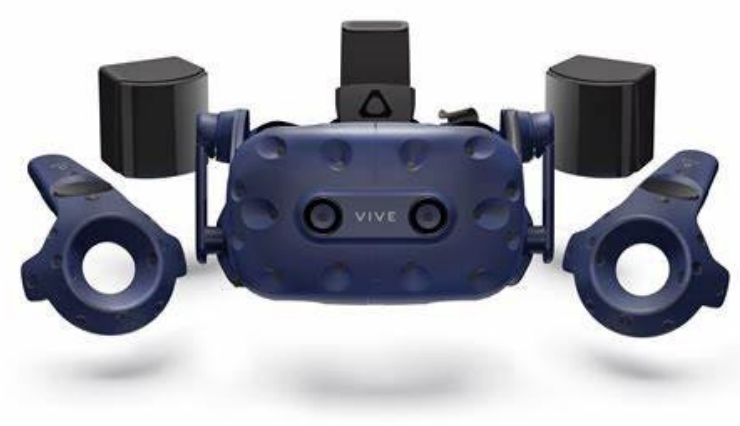

Figure 1 - VR Equipment-VIVE Pro

VR scene is built according to real dimension of the triple patients ward with new adding lighting equipment: two LED panels lamps on the ceiling, size $300 \mathrm{~mm} * 1200 \mathrm{~mm}$, CCT $3000 \mathrm{~K}-6000 \mathrm{~K}$ adjustable, Brightness $0-100 \%$ adjustable; three bedside wall lamps, size $200 \mathrm{~mm} * 1000 \mathrm{~mm}$, up and down light, brightness $0-100 \%$ adjustable; three Media interface lamps, installed on the bed end wall, is on each bed tail, size $300 \mathrm{~mm}^{*} 600 \mathrm{~mm}, \mathrm{RGB}$ change, brightness $0-100 \%$ adjustable. 


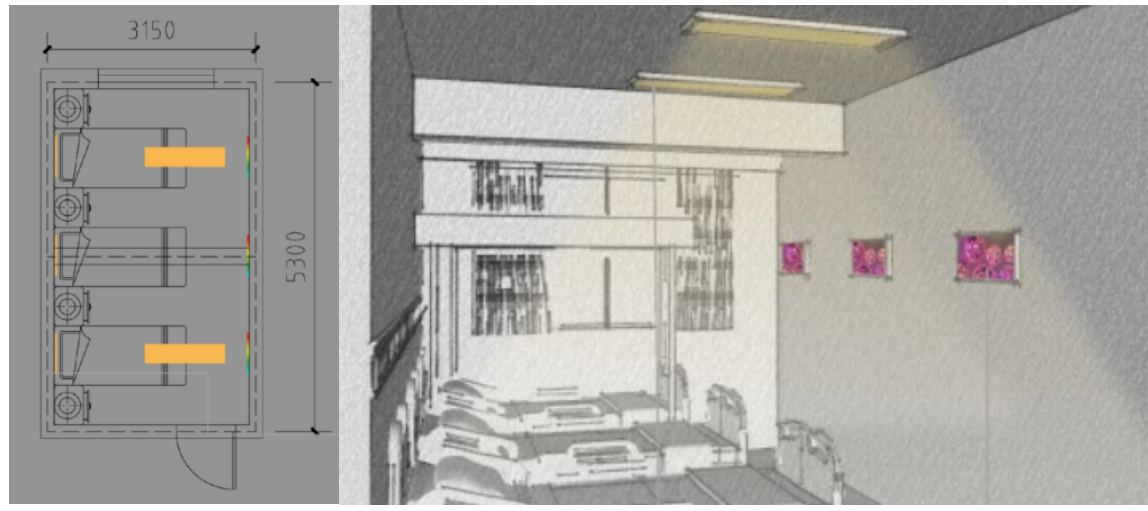

Figure 2 - Sketches of Patient Ward

\subsection{Subjects}

Six ( 3 male and 3 female) patients with blood cancer who were hospitalized between March and April 2019 were selected, with the minimum age of 19 and the maximum age of 70 . Six (1 male and 5 female) medical staff aged 25 45 working in the Haematology Department were selected. It was understood that the subjects had no eye disease or colour blindness before the experiment. The experiment allowed the patients to wear glasses to meet their normal visual needs. The purpose and content of the experiment were informed to patients and their families before the experiment and the experiment was conducted with their consent. As the subjects wore VR devices, the experimenter read out the questionnaire and recorded for the subjects.

\subsection{Design and Procedure}

\subsubsection{Experiment on environmental colour preference}

The experimenter put on the VR helmet and began the experiment after the subjects adapted. 12 kinds of wall and curtain colour sets were designed in the experiment scenes. The experimenter operated the computer and asked the subjects to look at all the scenes first, so that the subjects could have a comprehensive tour, and then switch each scene, and ask the subjects to score $(1-5 ; 1$ : the least favourite, and 5 : the most favourite), and finally choose the favourite colour.

The principle of colour setting uses the matching principle of high short tone $(B=9: 7)$ and soft hue $(S<30 \%)$, colour selection according to wall Hue drafted method, $\mathrm{H}=15,45,75,105,135,165,195,225,255,285,315,345$ and $\mathrm{S}=15 \%, \mathrm{~B}=90 \%$ collocation, as well as curtain $\mathrm{h}=15,45,75,105,135,165,195,225,285,315,345$, paired with $\mathrm{S}=24 \%, \mathrm{~B}=70 \%$ settings.
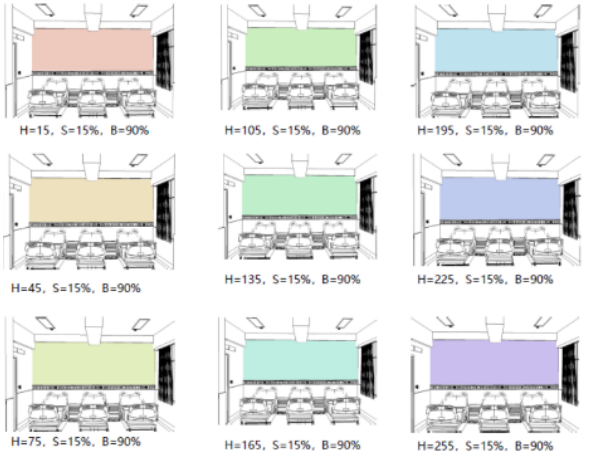
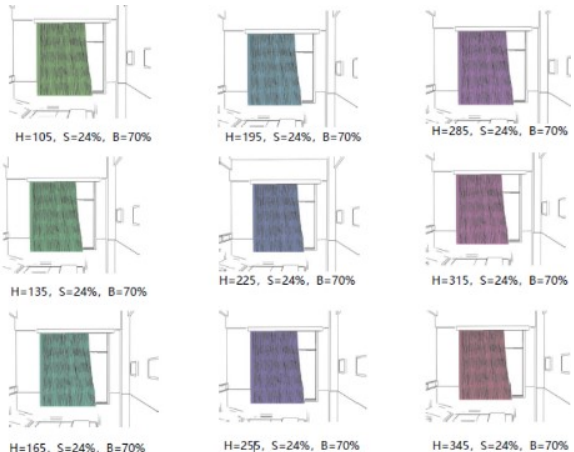

$H=345,5=24 \%, 8=70 \% 5$

Figure3 - Colour Selections

\subsubsection{Experiment on lighting scene}

The ward lighting scene experiment was carried out according to the favourite colour identified by the subjects. Based on the pre-investigation of the patient needs, six scenes were pre-set. scene 1 for the morning lighting scene, a total of three options (A. Colour temperature 6500K, bed illumination $1000 \mathrm{~lx}$, colour; B. Colour temperature $6500 \mathrm{~K}$, bed illumination $900 \mathrm{~lx}$; C. Colour temperature $4000 \mathrm{~K}$, bed illumination $1000 \mathrm{~lx}$;); scene 2 is a daily scene, with a total of three 
options: A. Colour temperature 4000K, bed illumination 300Lx;B. Colour temperature 4000K, bed illumination 500Ix; C. Colour temperature $3000 \mathrm{~K}$, bed illumination $300 \mathrm{~lx}$; scene 3 is bedtime light scene: A. Colour temperature $2700 \mathrm{~K}$, bed illumination 50Lx;B. Colour temperature $2700 \mathrm{~K}$, bed illumination 100lx; C. Colour temperature 4000K, bed illumination 20lx; Scene 4 is daytime check scene, A total of three scene selection: A. Colour temperature $5000 \mathrm{~K}$, bed illumination 500Lx;B. Colour temperature 5000K, bed illumination 900lx; C. Colour temperature 4000K, bed illumination $7001 x$; Scene 5 is a reading scene, A total of three scene selection: A. Colour temperature $4000 \mathrm{~K}$, bed illumination $400 \mathrm{Lx}$; B.Colour temperature $4000 \mathrm{~K}$, bed illumination $700 \mathrm{Ix}$; C. Colour temperature $3000 \mathrm{~K}$, bed illumination 400Ix; Scene 6 is a late-night check scene, A total of three scene selection: A. Colour temperature $4000 \mathrm{~K}$, bed illumination $300 \mathrm{Lx} ; \mathrm{B}$. Colour temperature $4000 \mathrm{~K}$, bed illumination $500 \mathrm{Ix}$; C. Colour temperature $3000 \mathrm{~K}$, bed illumination $2001 x$.

\section{Results}

\subsection{Colour Preferences}

By analysing the patient's favourite indoor colour is light blue $(H=195, S=15 \%, B=90 \%)$, while the indoor colour of the medical care preference is light yellow $(\mathrm{H}=45, \mathrm{~S}=15 \%, \mathrm{~B}=90 \%)$. In total, colour light yellow is chosen for the best one.

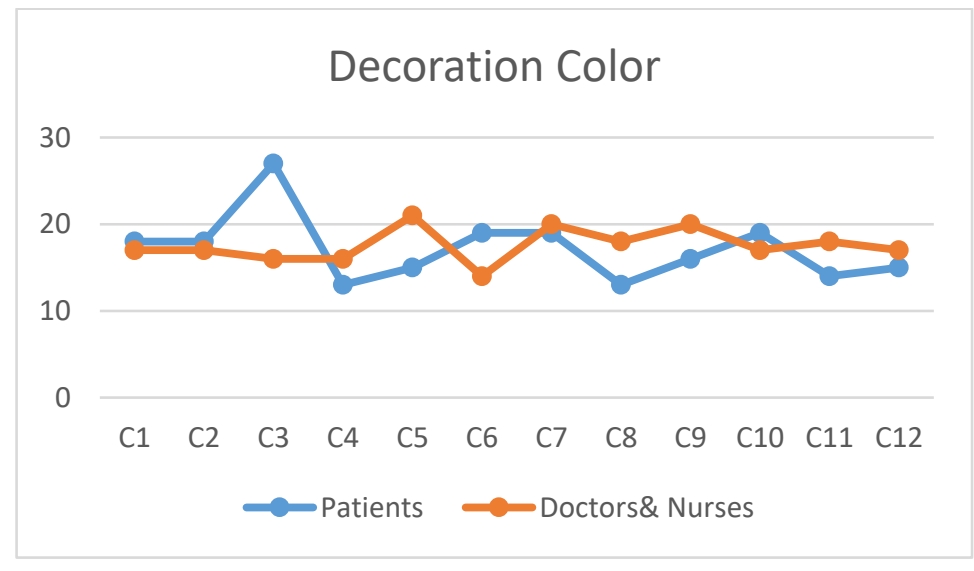

Figure 4 - Decoration Colour

\subsection{Scene Preferences}

Through data analysis, the patient prefers that the Morning lighting scene (CCT $=4000 \mathrm{~K}$, $E=10001 x)$, check the scene $(C C T=5000 K, E=9001 x ; C C T=4000 K, E=7001 x)$, Reading scene $(C C T=3000 K, E=4001 x)$, Daily scene $(C C T=4000 K, E=5001 x)$, Pre-bedtime lighting scene $(C C T=2700 K, E=501 x ; C C T=2700 K, E=1001 x ; C C T=4000 K, E=201 x)$, Late-night check scene $(\mathrm{CCT}=4000 \mathrm{~K}, \mathrm{E}=300 \mathrm{~lx}, \mathrm{CCT}=3000 \mathrm{~K}, \mathrm{E}=200 \mathrm{~lx})$ combined to form a 24-hour dynamic lighting model of the haematology ward, can meet the patient's rehabilitation environment requirements. Medical care think morning lighting scene $(C C T=4000 K, E=1000 \mathrm{Ix})$, Checking scene $(C C T=5000 K, E=9001 x)$, reading scene $(C C T=3000 K, E=4001 x)$, Daily scene $(C C T=4000 K$, $\mathrm{E}=500 \mathrm{~lx})$, Pre-bedtime lighting scene $(\mathrm{CCT}=4000 \mathrm{~K}, \mathrm{E}=20 \mathrm{~lx})$, Late-night check scene $(\mathrm{CCT}=4000 \mathrm{~K}, \mathrm{E}=300 \mathrm{~lx}, \mathrm{CCT}=3000 \mathrm{~K}, \mathrm{E}=200 \mathrm{~lx})$ combined to form a 24-hour dynamic lighting model of the haematology ward, to meet the requirements of the haematology doctor's work. 


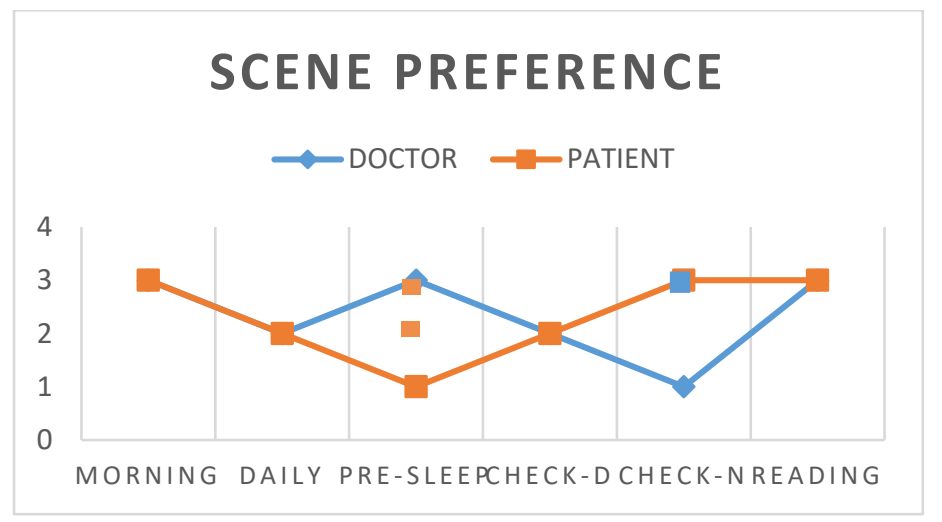

Figure 5 - Scene preferences

In comprehensive consideration of patient and medical data analysis, Morning lighting scene $(C C T=4000 K, E=1000 \mathrm{Ix})$, Check scene $(C C T=5000 \mathrm{~K}, E=900 \mathrm{~lx})$, Reading scene $(\mathrm{CCT}=3000 \mathrm{~K}$, $E=4001 x)$, Daily scene $(C C T=4000 K, E=5001 x)$, Pre-bedtime lighting scene (CCT $=4000 \mathrm{~K}$, $\mathrm{E}=201 \mathrm{x})$, Late-night check scene $(\mathrm{CCT}=4000 \mathrm{~K}, \mathrm{E}=300 \mathrm{Ix}, \mathrm{CCT}=3000 \mathrm{~K}, \mathrm{E}=200 \mathrm{~lx})$ combined to form a 24-hour dynamic lighting model of the haematology ward, It can not only meet the requirements of the haematology doctor's fine visual operation, but also bring the most suitable rehabilitation environment for the patient, and relieve the depressed mood of the patients.

\section{Discussion}

\subsection{Colour preferences and Emotion}

Colour psychology research shows that certain colours can exert different degrees of psychological adjustment on various types of patients. For example, red can promote blood circulation, rejuvenate, stimulate emotions, and have a certain stimulating effect on patients with depression. Blue can lower blood pressure and relax muscles, which helps manic patients to calm their emotions and psychology. It may explain why patients prefers the blue, blue has the effect of soothing.

Another thing is that Colour Combining lighting with nature elements would greatly alleviating people's anxiety, break the cold medical atmosphere and bring vitality to patients. The element installed on the bed opposite wall helps patient to relax, especially for those in laminar flow bed.

\subsection{Scene preferences}

The lighting scene setting was based on the interview records and investigation of the lives of doctors, nurses and patients. It respected the life and treatment needs of space users, and combined with the setting of scenes that were conducive to the health of patients in lighting.

The setting of morning getting-up scene was helpful to the patients' rhythm repair. Patients with blood cancer usually feel weak and have low immunity. They rarely go out of the ward to directly contact with natural light and spend most of their time in bed treatment or rest in the ward. At the same time, anxiety and depression of patients with blood cancer may cause insomnia. According to the field survey, about $17 \%$ of the patients had poor sleep, and $38 \%$ suffered from insomnia and nausea due to side effects of treatment. The present ward had was deeper (about $5.3 \mathrm{~m}$ ), and daylight was relatively abundant near the window during daytime, but it was unstable. Based on the biological effect of light, a morning getting-up lighting scene was established to help the patients with rhythm repair. The factors influencing rhythm regulation included light intensity, action time point, action duration and spectral power distribution (SPD). In VR scenes, patients were expected to compare the combination of light intensity and colour temperature, and the action duration and spectral power distribution were reserved for future experiments. In the scene, the illumination was set as $1000 \mathrm{~lx}, 900 \mathrm{~lx}, 1500 \mathrm{~lx}$, and the colour temperature was selected as $6500 \mathrm{~K}$ and $3000 \mathrm{~K}$. Meanwhile, the scene could provide sufficient illumination for medical examination when patients got up in the morning. 
Blood cancer is mainly treated through infusion chemotherapy, and fine drug dispensing by combining with the patients' physical condition and disease. First of all, the patients' physical condition needed to be strictly monitored, and had daily blood sampling, urine testing, cardiac monitoring, bone piercing and other tests. Through the feedback of the changes in the physical condition, it was vital to timely adjust the medication program. Secondly, drug administration time should be precisely controlled. At present, the treatment options in the hospital was mainly divided into 9393 infusion method and 951 infusion method. To be specific, infusion was applied at 9:00AM, 3:00PM, 9:00PM, 3:00AM or drugs were given at 9:00AM, 5:00PM, and 1:00AM. Therefore, according to the needs of medical staff for examination at different times, day examination and late night scene were set. If the curtains were open in the daytime during patients' rest, and lights were turned off at night, they could not meet the needs of medical staff for examination and treatment. Working lighting was provided for indoor examination by bedside wall lamp and ceiling lamp. Infusion and dressing should be completed with less interference on other patients in the ward as far as possible especially in the night.

Laminar Flow bed provides a local cleansing environment for patients who have just had low immunity from chemotherapy, helping to control the patient's body temperature and reduce secondary infection. The fan in the above is open 24 hours a day, with high noise is about 3850 decibels, and the patient has a strong sense of isolation in it. Coupled with the patient's own weakness, their negative emotions are serious. The way to relax during treatment is mostly to read books or electronic screens chosen by blood cancer patients, so that individual reading scenes was set to meet the needs of this part of patients.

\subsection{VR Simulation}

The optical environment model in this study uses the Unreal 4 software based on PBR technology to construct and render of the basic model. The methods and algorithms of PBR technology are based on accurate calculation formulas, which has direct connection input parameters and final optical results, would be competent for the impact of multiple variables of complex light environment without losing truth. It provides open and unified interface which can access optical data files, act real simulation of illumination with brightness of the data in consideration of different variables in the light environment, such as lighting, natural light and interior decoration. All of above factors influenced can draw the accurate conclusion of calculation. The PBR method is basically the same as the Dialux Light environment simulation principle commonly used in the field of light environment, which is realized by photon mapping by using the built-in IES light parameters and RGB chromaticity parameters. However the former one rendering effect is better, and the embodiment and cognition of the light environment can be more accurate than that of Dialux. So that accurate simulation of brightness and chroma can be achieved. Coupled with the final scene of the light environment through VR technology to the subjects presented, the combination of the two, can better ensure that the scene effect and the relevant conclusions are obtained.

\section{Conclusion}

This study tries the experiment method of VR and set tailored lighting scenes for doctors and patients. It finally gives the satisfying lighting scenes to alleviate doctors and patients' mood, and provides guidance for practical design application:

1 Room lighting environment tuning over time can improve patients' satisfaction.

2 Treatment scenes should be divided into day and night modes, especially treatment mode is required in the late night, to minimize the impact on patients' rest at night

3 Soft hue and a high short collocation of colour in the ambient environment is suitable for hospital.

\section{Funding}

National Key Research and Development Program of China (No. 2017YFB0403704). 


\section{References}

1. An, N. 1999. Colour Principle and Colour Composition, Zhejiang Academy of Fine Arts Publishing House.

2. Hamilton, D. K, Bai, X. 2012. Evidence-based design: guiding hospital design with scientific research [J]. Chinese Hospital Architecture and Equipment, 13 (10): 37-41.

3. JOSEPH, A., HAMILTON. D. K.. 2008. The Pebble Projects: Coordinated Evidence-based Case Studies [J]. Building Research and Information, 2,129-145.

4. Li, H.J. et al.2009. Virtual Reality Art: Metaphysical Ultimate Recreation. Art Today, 04, 17-23.

5. $\mathrm{Li}, \mathrm{P}$. et al. 2017. Teaching Practice of PBL+VR in the Course of Hospital Management Information System. New Curriculum Research, 10, 38-39

6. Lu, Z. Zhu, X. 2012.Theoretical research and practice of evidence-based design [J]. Chinese Hospital Architecture and Equipment, 10, 24-29.

7. WU, Z.L., et al. 2017. "VR + Medical" Pioneer. Career Development, Z1, 12-16

8. QIU, H.L., et al. 2019. The application value of virtual reality technology in the surgical treatment of coronary artery fistula and abnormal origin of coronary artery. Chinese Journal of Clinical Thoracic and Cardiovascular Surgery, 03,217-221

9. Wu, X.W., et al. 2007. Application Prospect of Virtual Reality Technology (VR) in Medical Treatment. Clinical Medicine \& Engineering, 05, 17-20

10. Zeng, Q.X., et al. 2018. Personal Priority Effect in Personal Space Perception [A]. Chinese Psychological Association. Summary of the 21st National Psychological Conference [C] 